\title{
JULIA SETS ARE UNIFORMLY PERFECT
}

\author{
R. MAÑÉ AND L. F. DA ROCHA
}

(Communicated by Kenneth R. Meyer)

\begin{abstract}
We prove that Julia sets are uniformly perfect in the sense of Pommerenke (Arch. Math. 32 (1979), 192-199). This implies that their linear density of logarithmic capacity is strictly positive, thus implying that Julia sets are regular in the sense of Dirichlet. Using this we obtain a formula for the entropy of invariant harmonic measures on Julia sets. As a corollary we give a very short proof of Lopes converse to Brolin's theorem.
\end{abstract}

Let $\overline{\mathbf{C}}$ be the Riemann sphere. As usual we say that a set $A \subset \overline{\mathbf{C}}$ is an annulus if there exists, for some $0<r<1$, a conformal representation of $\{z \in \mathbf{C}|r<| z \mid<1\}$ onto $A$. The number $\log (1 / r)$ is called the modulus of $A$.

Given a set $K \subset \overline{\mathbf{C}}$, we say that an annulus $A$ divides $K$ if $K \cap A=\varnothing$ and $K$ intersects both connected components of the complement $A^{c}$ of $A$.

In [6] Pommerenke introduced the following definition: a set $K \subset \overline{\mathbf{C}}$ is said to be uniformly perfect if it contains more than one point and there exists $m>0$ such that every annulus that divides $K$ has modulus $\leq m$. In particular, connected sets satisfy this definition since no annulus can divide them thus making the condition vacuous.

A uniformly perfect set $K$ is always regular (in the sense of Dirichlet), i.e., for every continuous function $\varphi: K \rightarrow \mathbf{R}$ there exists a continuous function $\varphi^{*}: \overline{\mathbf{C}} \rightarrow$ $\mathbf{R}$ such that $\varphi^{*} / K=\varphi$ and $\varphi$ is harmonic in $K^{c}$. The function $\varphi^{*}$ (that is unique) will be called the harmonic extension of $\varphi$. In fact Pommerenke proved in [6] a much stronger property, namely, that denoting $d(\cdot, \cdot)$ the spherical metric on $\overline{\mathbf{C}}$ and denoting $\gamma(S)$ the logarithmic capacity of a compact set $S$, a compact set $K \subset \overline{\mathbf{C}}$ is uniformly perfect if and only if there exists $\delta>0$ such that for all $a \in K$ and $r>0$

$$
\gamma(\{z \in K \mid d(z, a) \leq r\}) \geq \delta r .
$$

It is well known that this property implies the regularity of $K$ (see, for instance, [8]).

Our first objective is to prove the following result that answers positively a question posed by Pommerenke in [7]. Afterwards we shall apply it to give a formula for the entropy of harmonic measures on Julia sets through which we shall recover, with much shorter proofs, some already known results relating

Received by the editors December 20, 1990.

1991 Mathematics Subject Classification. Primary 58F11; Secondary 31A15. 
harmonic measures with the maximizing (i.e., the entropy maximizing) measure of a rational map.

Theorem. The Julia set $J(f)$ of a rational map $f: \overline{\mathbf{C}} \hookleftarrow$ is uniformly perfect.

Proof. Suppose, by contradiction, that there is a sequence of annuli $A_{n}, n=$ $1,2, \ldots$, dividing $K$ such that $\lim _{n \rightarrow+\infty} \bmod \left(A_{n}\right)=\infty$. This property implies that for each $n$, a connected component $K_{n}$ of $A_{n}^{c}$ can be chosen so that $\lim _{n \rightarrow+\infty} \operatorname{diam} K_{n}=0$. Denote by $K_{n}^{\prime}$ the other connected component of $A_{n}^{c}$. Then inf ${ }_{n>0}$ diam $K_{n}^{\prime}>0$ because otherwise we could take a subsequence $\left\{K_{n_{j}}^{\prime}\right\}$; with $\lim _{j \rightarrow+\infty} \operatorname{diam} K_{n_{j}}^{\prime}=0$ and points $p_{j}^{\prime} \in K_{n_{j}}^{\prime} \cap J(f)$, $p_{j} \in K_{n} \cap \cap(f)$ converging to points $p^{\prime}$ and $p$ in $J(f)$, and then, from $\lim _{j \rightarrow+\infty} \operatorname{diam} K_{n_{j}}^{\prime}=\lim _{j \rightarrow+\infty} \operatorname{diam} K_{n_{j}}=0$ and $J(f) \subset K_{n_{j}} \cup K_{n_{j}}^{\prime}$ for all $j$, it follows that $J(f)=\{p\} \cup\left\{p^{\prime}\right\}$, which is impossible. Denote $D=\{z \in \mathbf{C}|| z \mid<$ $1\}$ and let $\varphi_{n}: D \rightarrow A_{n} \cup K_{n}$ be a conformal representation with $\varphi_{n}(0) \in K_{n}$. Then $\bmod \left(D-\varphi_{n}^{-1}\left(K_{n}\right)\right)=\bmod \left(A_{n}\right)$. Hence $\lim _{n \rightarrow+\infty} \operatorname{diam} \varphi_{n}^{-1}\left(K_{n}\right)=0$ because $\lim _{n \rightarrow+\infty} \bmod \left(A_{n}\right)=\infty$. Take $1>r_{n}>\rho_{n}>2 \operatorname{diam}\left(K_{n}\right)$ satisfying $\lim _{n \rightarrow+\infty} r_{n}=0, \lim _{n \rightarrow+\infty} \rho_{n} / r_{n}=0$. Set $D_{n}^{\prime}=\left\{z|| z \mid<\rho_{n}\right\}$. The family of functions $\varphi_{n}: D \rightarrow \overline{\mathbf{C}}$ is normal because $\inf _{n} \operatorname{diam}_{\varphi_{n}}(D)^{c}=\inf _{n} \operatorname{diam} K_{n}^{\prime}>0$. Hence $\lim _{n \rightarrow+\infty} \operatorname{diam} \varphi_{n}\left(D_{n}^{\prime}\right)=0$. But $\varphi_{n}\left(D_{n}^{\prime}\right)$ is an open set containing points of $J(f)$. Therefore the classical theory of Julia sets implies that there exist integers $t_{n}>0$ such that $f^{t_{n}}\left(\varphi_{n}\left(D_{n}^{\prime}\right)\right) \supset J(f)$. Take $0<c<\operatorname{diam} J(f)$, and let $m_{n}$ be the minimum positive integer such that $\operatorname{diam} f^{m_{n}}\left(\varphi_{n}\left(D_{n}^{\prime}\right)\right) \geq c$. Since $\lim _{n \rightarrow+\infty} \operatorname{diam} \varphi_{n}\left(D_{n}^{\prime}\right)=0$, it follows that $\lim _{n \rightarrow+\infty} m_{n}=+\infty$. Moreover, since $\operatorname{diam} f^{m_{n}-1}\left(\varphi_{n}\left(D_{n}^{\prime}\right)\right)<c$, it follows that diam $f^{m_{n}}\left(\varphi_{n}\left(D_{n}^{\prime}\right)\right)<L c$, where $L$ is the Lipschitz constant of $f$. Let $S$ be a set of four different points in $J(f)$. Take $c$ so small that every set of diameter $\leq L c$ cannot contain two of them. Then $f^{m_{n}}\left(\varphi_{n}\left(D_{n}^{\prime}\right)\right)$ does not cover three points of $S$. Define $\psi_{n}: D \rightarrow \overline{\mathbf{C}}$ by $\psi_{n}(z)=f^{m_{n}} \varphi_{n}\left(r_{n} z\right)$. Let us show that the family $\left\{\psi_{n}\right\}$ is normal. It suffices to show that for all $n, \psi_{n}(D)$ does not cover three points (that may depend on $n$ ) of $S$. If $\rho_{n} / 2 r_{n}<|z|<1$ then $\rho_{n} / 2<\left|r_{n} z\right|<r_{n}$ and, since $\operatorname{diam} \varphi_{n}^{-1}\left(K_{n}\right) \leq \rho_{n} / 2$, it follows that $r_{n} z \notin \varphi_{n}^{-1}\left(K_{n}\right)$ and $\varphi_{n}\left(r_{n} z\right) \notin K_{n}$. Then $\varphi_{n}\left(r_{n} z\right) \notin J(f)$ because $K_{n}=J(f) \cap \varphi_{n}(D)$. Hence $\psi_{n}(z)=f^{m_{n}} \varphi_{n}\left(r_{n} z\right) \notin$ $f^{m_{n}}(J(f))=J(f)$. Therefore $\psi_{n}(z) \notin S$ when $\rho_{n} / 2 r_{n}<|z|<1$. On the other hand, if $|z| \leq \rho_{n} / 2 r_{n}$, it follows that $\left|r_{n} z\right| \leq \rho_{n} / 2 \leq \rho_{n}$ and then $\psi_{n}(z)=f^{m_{n}} \varphi_{n}\left(r_{n} z\right) \in f^{m_{n}} \varphi_{n}\left(D_{n}^{\prime}\right)$ that does not cover three points of $S$. This proves the normality of the family $\left\{\psi_{n}\right\}$. Then, given $\varepsilon>0$, there exists a neighborhood $V$ of 0 such that diam $\psi_{n}(V) \leq \varepsilon$ for all $n$. But for $n$ sufficiently large, $V \supset\left\{z|| z \mid<\rho_{n} / r_{n}\right\}$.

$$
\begin{aligned}
\varepsilon & \geq \operatorname{diam} \psi_{n}(V) \geq \operatorname{diam} \psi_{n}\left(\left\{z|| z \mid<\rho_{n} / r_{n}\right\}\right) \\
& =\operatorname{diam} f^{m_{n}} \varphi_{n}\left(D_{n}^{\prime}\right) \geq c .
\end{aligned}
$$

Since $\varepsilon>0$ is arbitrary, this is a contradiction that proves the theorem.

Let us show some applications of the regularity of the Julia set. Recall that, given a regular compact set $K \subset \overline{\mathbf{C}}$ and a point $p \in \overline{\mathbf{C}}$, the harmonic measure $\mu_{p}$ is defined as the probability on the Borel $\sigma$-algebra of $K$ such that the integral with respect to $\mu_{p}$ of a continuous function $\varphi: K \rightarrow \mathbf{R}$ is given by

$$
\int \varphi d \mu_{p}=\varphi^{*}(p)
$$


where $\varphi^{*}: \overline{\mathbf{C}} \rightarrow \mathbf{R}$ is the harmonic extension of $\varphi$. If $p \notin K$ the support of $\mu_{p}$ is obviously the boundary of the connected component of $K^{c}$ that contains $p$, and it is well known that if $p$ and $q$ are in the same connected component of $K^{c}$ then $\mu_{p}$ and $\mu_{q}$ are equivalent and the Radon-Nykodim derivative $d \mu_{p} / d \mu_{q}$ is bounded and has a strictly positive infimum.

Observe that if $f: \overline{\mathbf{C}} \hookleftarrow$ is a rational map and $\varphi: J(f) \rightarrow \mathbf{R}$ is continuous, then $(\varphi \circ f)^{*}=\varphi^{*} \circ f$ because $\varphi^{*} \circ f$ is harmonic on the complement of $J(f)$ and $\left(\varphi^{*} \circ f\right)|J(f)=(\varphi \circ f)| J(f)$. Then

$$
\int(\varphi \circ f) d \mu_{p}=\int \varphi d \mu_{f(p)}
$$

because

$$
\int(\varphi \circ f) d \mu_{p}=(\varphi \circ f)^{*}(p)=\left(\varphi^{*} \circ f\right)(p)=\varphi^{*}(f(p))=\int \varphi d \mu_{f(p)} .
$$

Hence, $\mu_{p}$ is $f$-invariant if and only if $f(p)=p$.

Given an attracting fixed point $p$ of $f$, define its basin $W^{s}(p)$ as the set of points $z$ such that $\lim _{n \rightarrow+\infty} f^{n}(z)=p$ and its immediate basin $B^{s}(p)$ as the connected component of $W^{s}(p)$ that contains $p$. Then $\mu_{p}$ is an invariant probability of $f$ whose support is the boundary $\partial B^{s}(p)$ of $B^{s}(p)$.

Corollary 1. If $f: \overline{\mathbf{C}} \hookleftarrow$ is a rational map and $p$ is an attracting fixed point of $f$, the entropy $h_{\mu_{p}}(f)$ of $f$ with respect to $\mu_{p}$ is given by

$$
h_{\mu_{p}}(f)=\int \log \left(\sum_{x \in f^{-1}(p) \cap B^{s}(p)} \frac{d \mu_{x}}{d \mu_{p}}\right) d \mu_{p}
$$

where the points $x \in f^{-1}(p)$ are repeated according to its multiplicity.

Proof. Define $J: \partial B_{s}(p) \rightarrow \mathbf{R}$ by

$$
J=\sum_{x \in f^{-1}(p) \cap B^{s}(p)} \frac{d \mu_{x}}{d \mu_{p}} .
$$

Let us prove that $J$ is the Jacobian of $\mu_{p}$, i.e., that

$$
\mu_{p}(f(A))=\int_{A} J d \mu_{p}
$$

for every Borel set $A \subset \partial B^{s}(p)$ such that $f / A$ is injective. Once this is proved, the corollary follows from the formula

$$
h_{\mu_{p}}(f)=\int \log J d \mu_{p}
$$

proved in [5]. To prove (1) denote $C^{0}\left(\partial B^{s}(p)\right)$ and $C^{0}\left(\overline{B^{s}(p)}\right)$ the spaces of continuous functions of $\partial B^{s}(p)$ and $\overline{B^{s}(p)}$ on $\mathbf{R}$ endowed with the norm of the supremum. Given a function $\varphi: \partial B^{s}(p) \rightarrow \mathbf{R}\left(\right.$ resp. $\left.\varphi: B^{s}(p) \rightarrow \mathbf{R}\right)$, define $\mathscr{L}(\varphi): \partial B^{s}(p) \rightarrow \mathbf{R}$ (resp. $\mathscr{L}(\varphi): B^{s}(p) \rightarrow \mathbf{R}$ ) by $\mathscr{L}(\varphi)(x)=\sum_{y} \varphi(y)$ where the sum is taken over all the $y$ 's in $f^{-1}(x) \cap \partial B^{s}(p)$ (resp. $y \in f^{-1}(x) \cap$ $\left.\overline{B^{s}(p)}\right)$ repeated according to its multiplicity. Observe that $\mathscr{L}$ maps continuous functions in continuous functions. Then if $\varphi \in C^{0}\left(\partial B^{s}(p)\right)$ then the harmonic extension $(\mathscr{L}(\varphi))^{*} \in C^{0}\left(\overline{B^{s}(p)}\right)$ of $\mathscr{L}(\varphi)$ satisfies $(\mathscr{L}(\varphi))^{*}=\mathscr{L}\left(\varphi^{*}\right)$. To see 
this observe that $\mathscr{L}\left(\varphi^{*}\right)$ is harmonic in the complement of the critical values of $f$ because there it is locally the sum of the harmonic function $\varphi^{*}$ composed with the holomorphic branches of $\left(f \mid B^{s}(p)\right)^{-1}$. Moreover $\mathscr{L}\left(\varphi^{*}\right)$ is obviously continuous; hence $\mathscr{L}\left(\varphi^{*}\right)$ is harmonic. Clearly we have $\mathscr{L}\left(\varphi^{*}\right) / \partial B^{s}(p)=$ $\mathscr{L} \varphi$. Hence $\mathscr{L}\left(\varphi^{*}\right)=(\mathscr{L}(\varphi))^{*}$. Then, if $\varphi \in C^{0}\left(\partial B^{s}(p)\right)$,

$$
\begin{aligned}
\int \mathscr{L}(\varphi) d \mu_{p} & =(\mathscr{L}(\varphi))^{*}(p)=\mathscr{L}\left(\varphi^{*}\right)(p) \\
& =\sum_{x \in f^{-1}(p) \cap B^{s}(p)} \varphi^{*}(x)=\sum_{x \in f^{-1}(p) \cap B^{s}(p)} \int \varphi d \mu_{x} \\
& =\int \varphi\left(\sum_{x \in f^{-1}(p) \cap B^{s}(p)} \frac{d \mu_{x}}{d \mu_{p}}\right) d \mu_{p}=\int \varphi J d \mu_{p} .
\end{aligned}
$$

From this equality it follows, by standard methods, that

$$
\int \mathscr{L}(\varphi) d \mu_{p}=\int \varphi J d \mu_{p}
$$

for every bounded measurable $\varphi: \partial B^{s}(p) \rightarrow \mathbf{R}$. Apply it to the case when $\varphi$ is the characteristic function of a Borel set $A \subset \partial B^{s}(p)$ such that $f / A$ is injective. Then $\mathscr{L}(\varphi)$ is the characteristic function of $f(A)$. Hence

$$
\mu_{p}(f(A))=\int \mathscr{L}(\varphi) d \mu_{p}=\int \varphi J d \mu_{p}=\int_{A} J d \mu_{p}
$$

completing the proof of the corollary.

For the next corollary recall that $[4,2,5]$ if $\operatorname{deg}\left(f / B^{s}(p)\right)$ denotes the degree of $f / B^{s}(p)$ (i.e., the number of preimages in $B^{s}(p)$ of any $x \in B^{s}(p)$ counted with multiplicity) then the topological entropy of $f \mid \partial B^{s}(p)$ is $\log \operatorname{deg}\left(f \mid B^{s}(p)\right)$ and there exists a unique probability $\mu$ on $\partial B^{s}(p)$, invariant under $f \mid \partial B^{s}(p)$, such that $h_{\mu}(f)=\log \operatorname{deg}\left(f \mid B^{s}(p)\right)$.

Corollary 2. $h_{\mu_{p}}(f)=\log \operatorname{deg}\left(f \mid B^{s}(p)\right)$ if and only if $f^{-1}(p) \cap B^{s}(p)=p$.

Proof. If $f^{-1}(p) \cap B^{s}(p)=p$ then the formula of Corollary 1 immediately implies $h_{\mu_{p}}(f)=\log \operatorname{deg} B^{s}(p)$ because $d \mu_{x} \mid d \mu_{p} \equiv 1$ for all $x \in f^{-1}(p) \cap B^{s}(p)$. To prove the converse property observe that by Jensen inequality

$$
\begin{aligned}
h_{\mu_{p}}(f) & =\int \log \left(\sum_{x \in f^{-1}(p) \cap B^{s}(p)} \frac{d \mu_{x}}{d \mu_{p}}\right) d \mu_{p} \\
& \leq \log \int\left(\sum_{x \in f^{-1}(p) \cap B^{s}(p)} \frac{d \mu_{x}}{d \mu_{p}}\right) d \mu_{p}=\log \sum_{x \in f^{-1}(p) \cap B^{s}(p)}\left(\int d \mu_{x}\right) \\
& =\log \operatorname{deg}\left(f \mid B^{s}(p)\right)
\end{aligned}
$$

and the equality holds if and only if

$$
\sum_{x \in f^{-1}(p) \cap B^{s}(p)} \frac{d \mu_{x}}{d \mu_{p}}=\operatorname{deg}\left(f \mid B^{s}(p)\right)
$$


$\mu_{p}$-almost everywhere. Denote $m=\operatorname{deg}\left(f \mid B^{s}(p)\right)$ and let $S$ be the set of points in $f^{-1}(p) \cap B^{s}(p)$ repeated according to its multiplicity. Then

$$
m \varphi^{*}(p)=\sum_{x \in S} \varphi^{*}(x)
$$

for every $\varphi \in C^{0}\left(\partial B^{s}(p)\right)$ because

$$
\begin{aligned}
m \varphi^{*}(p) & =\int m \varphi d \mu_{p}=\int \varphi\left(\sum_{x \in S} \frac{d \mu_{x}}{d \mu_{p}}\right) d \mu_{p} \\
& =\sum_{x \in S} \int \varphi d \mu_{x}=\sum_{x \in S} \varphi^{*}(x) .
\end{aligned}
$$

Let us show that (2) implies that $x \in S$ implies $x=p$. Without loss of generality we shall assume that $\infty \in J(f)$. First we shall prove that (2) implies

$$
p=\frac{1}{m} \sum_{x \in S} x .
$$

Suppose that this is false. Then we can take a linear function $\psi: \mathbf{R}^{2}=\mathbf{C} \rightarrow \mathbf{R}$ such that

$$
\psi(p) \neq \frac{1}{m} \sum_{x \in S} \psi(x) .
$$

Given integers $n>0, k>0$, define $\varphi_{n, k} \in C^{0}\left(\partial B^{s}(p)\right)$ by

$$
\begin{array}{ll}
\varphi_{n, k}(z)=\psi(z) & \text { when }-k<\psi(z)<n, \\
\varphi_{n, k}(z)=n & \text { when } \psi(z) \geq n, \\
\varphi_{n, k}(z)=-k & \text { when } \psi(z) \leq-k .
\end{array}
$$

Then for each $k,\left\{\varphi_{n, k}\right\}_{n}$ is an increasing sequence and for each $n,\left\{\varphi_{n, k}\right\}_{k}$ is a decreasing sequence. Hence the same properties hold for $\left\{\varphi_{n, k}^{*}\right\}_{n},\left\{\varphi_{n, k}^{*}\right\}_{k}$. Then, using that $\psi$ is harmonic, it is easy to see that

$$
\psi=\lim _{k \rightarrow+\infty} \lim _{n \rightarrow+\infty} \varphi_{n, k}^{*}
$$

Then

$$
\varphi_{n, k}^{*}(p) \neq \frac{1}{m} \sum_{x \in S} \varphi_{n, k}(x)
$$

for $n, k$ sufficiently large, contradicting (2) and proving (3). But (3) implies

$$
T(p)=\frac{1}{m} \sum_{x \in S} T(x)
$$

for every Moebius map $T: \overline{\mathbf{C}} \hookleftarrow$ such that $T^{-1}(\infty) \in J(f)$ because $T f T^{-1}$ has $T(p)$ as an attracting fixed point whose preimages in this immediate basin are the points $\{T(x) \mid x \in S\}$ and $\infty \in J\left(T f T^{-1}\right.$ ) (by the property $T^{-1}(\infty) \in$ $J(f)$ ), and then, (3) implies (4). In particular (4) implies

$$
\frac{1}{p-z_{0}}=\frac{1}{m} \sum_{x \in S} \frac{1}{x-z_{0}}
$$


for all $z_{0} \in J(f)$ (taking $T$ as $T(z)=\left(z-z_{0}\right)^{-1}$ ). The number of values of $z_{0} \in \mathbf{C}$ for which this equality holds is either finite or holds for every $z_{0} \in \mathbf{C}$. In the first case, since $J(f)$ is infinite, we can take $z_{0} \in J(f)$ violating $(5)$ and thus proving Corollary 2 by contradiction. In the second case, the left and right sides of (5) are identical as functions of $z_{0} \in \mathbf{C}$. But the left side function has a unique pole at $z_{0}=p$, and the right side has poles at all the $x$ 's in $S$. Then, to be identical, we must have $p=x$ for all $x \in S$, proving the corollary.

Finally recall that a rational map $f: \overline{\mathbf{C}} \hookleftarrow$ of degree $d>1$ has topological entropy $\log d$ and a unique invariant probability $\mu_{\max }$ (the maximizing measure) for which $h_{\mu_{\max }}(f)=\log d([4,2,5])$. When $f$ is a polynomial, $\infty$ is an attracting fixed point and $f^{-1}(\infty)=\{\infty\}$ (with multiplicity $d$ ). Hence, by Corollary $1, h_{\mu_{\infty}}(f)=\log d$. We have thus proved the result of Brolin [1] stating that for polynomials the maximizing measure is the harmonic measure with respect to $\infty$. Using Corollaries 1 and 2 we can also prove the converse property (due to Lopes [3]): if for a rational map $f: \overline{\mathbf{C}} \hookleftarrow$ its maximizing measure coincides with its harmonic measure with respect to $\infty$ then $f$ is a polynomial. To prove this observe that $\mu_{\max }=\mu_{\infty}$ implies that $\mu_{\infty}$ is $f$-invariant. Hence $f(\infty)=\infty$. If $\infty \in J(f)$ then $\mu_{\infty}$ is the Dirac $\delta$ at $\infty$ and $h_{\mu_{\infty}}(f)$ would be 0 . Hence $\infty \notin J(f)$. Being a fixed point, the property $\infty \notin J(f)$ implies that it is either an attracting fixed point, or the center of a Siegel disk. In the first case, from Corollary 1 and Jensen's inequality, it follows that

$$
\log d=h_{\mu_{\infty}}(f) \leq \log \operatorname{deg}\left(f \mid B^{s}(\infty)\right) \leq \log d .
$$

Hence, all the equalities hold, implying that the number of points in $f^{-1}(\infty) \cap$ $B^{s}(\infty)$ counted with multiplicity is $d$ (because $\log \operatorname{deg}\left(f \mid B^{s}(\infty)\right)=\log d$ ) and, by Corollary 2, $f^{-1}(\infty) \cap B^{s}(\infty)=\{\infty\}$ (because $\log \operatorname{deg}\left(f \mid B^{s}(\infty)\right)=h_{\mu_{\infty}}(f)$ ). Since $f^{-1}(\infty)$ contains $d$ points (counted with multiplicity), it follows that $f^{-1}(\infty)=\{\infty\}$, which proves that $f$ is a polynomial. When $\infty$ is the center of a Siegel disk, $h_{\mu_{\infty}}(f)=0$. This follows, for instance, from observing that the formula in Corollary 1 holds also (without changing the proof) replacing $B^{s}(p)$ by a Siegel disk with fixed point $p$. But since in this case $f^{-1}(p)$ intersected with the Siegel disk contains only $p$, it follows that $h_{\mu_{p}}(f)=0$. Hence $h_{\mu_{\infty}}(f)=0$, obviously contradicting $h_{\mu_{\infty}}(f)=\log d$.

\section{REFERENCES}

1. H. Brolin, Invariant sets under iteration of rational functions, Ark. Mat. 6 (1965), 103-144.

2. A. Freire, A. Lopes, and R. Mañé, An invariant measure for rational maps, Bol. Soc. Brasil Mat. 14 (1983), 45-62.

3. A. Lopes, Equilibrium measures for rational maps, Ergodic Theory Dynamical Systems 6 (1986), 414-426.

4. M. Ju. Ljubich, Entropy properties of rational endomorphisms of the Riemann sphere, Ergodic Theory Dynamical Systems 3 (1983), 351-386.

5. R. Mañé, On the uniqueness of the maximizing measure for rational maps, Bol. Soc. Brasil Mat. 14 (1983), 27-43.

6. Ch. Pommerenke, Uniformly perfect sets and the Poincaré metric, Arch. Math. 32 (1979), 192-199. 
7. __ On uniformly perfect sets and Fuchsian groups, Analysis 4 (1986), 299-321.

8. M. Tsuji, Potential theory in modern function theory, Maruzen, Tokyo, 1959.

Instituto de Matemática Pura e Aplicada, Estrada Dona Castorina, 110 Jardim BotÂNICO, 22460 RIO DE JANEIRO-RJ, BRASIL

Instituto de Matemática, Universidade Federal do Rio Grande do Sul, Av. Bento Gonçalves, 9500, 91500 Porto Alegre-RS, Brasil 